\title{
Ink-Bleed Reduction using Layer Separation
}

\author{
Shrikant Baronia and Anoop Namboodiri \\ Internation Institute Of Information Technology Hyderabad, India \\ Email: shrikant.baronia@research.iiit.ac.in, anoop@iiit.ac.in
}

\begin{abstract}
We present a novel method for reducing the effects of ink-bleed in handwritten documents. We go beyond the existing works on ink bleed detection and removal. We consider each pixel in a document as a result of combination of foreground, ink-bleed and background. We carry of a decomposition of the document image into separate foreground ink, ink-bleed, and background layers. We propose an efficient MRF formulation to achieve this separation. Degradation model for the ink and paper is proposed. The ability to extract the contributions of the three components to each pixel allows us to recover finer details of the writing. Quantitative and qualitative results on a set of historic manuscripts as well as synthetically generated documents demonstrate the effectiveness of our approach.
\end{abstract}

\section{INTRODUCTION}

Historic handwritten documents are extremely useful in gaining valuable information regarding variety of topics including the specific subject of writing, societal aspects of the community that generated the document, language and script of the time, author of the document and writing medium. Unfortunately many of the documents undergo degradation from exposure to the environment as well as natural chemical processes within the paper and ink. These include discoloration of the paper, fading of the ink and ink bleed in addition to any direct damages caused by the elements, insects or humans. The former three are natural degradation that are difficult to avoid, while documents that are preserved well are not affected by the latter.

In this work, we aim to reduce the inkbleed from the document images by decomposing the document into ink, paper and bleed layers, as illustrated in Figure 1. In this respect, the current work is different from previous attempts at removal of ink bleed. The formulation also allows us to recover finer details of the ink and paper that are important in the analysis of documents for aspects such as authorship and script analysis. One can also find out the kind of writing instrument used, for example the quill pen made of crow feathers are best for making fine lines and goose quills were used for calligraphy writing.

Most of the previous work [1], [2], [3] focuses on classifying a pixel as either of foreground, ink bleed or background. In all these approaches the pixels labeled as ink bleed are converted to a background color, which is estimated by averaging selected background pixels. Such a binary classification results in loss of low level details and the background is averaged out completely. Instead of this discrete classification, we want to determine the amount of background, ink and inkbleed intensity at every pixel. The problem is inherently illposed as we have to recover the background values which are completely lost at many points. In addition, we would like to compute the amount of ink and ink-bleed components of pixels where both are present.

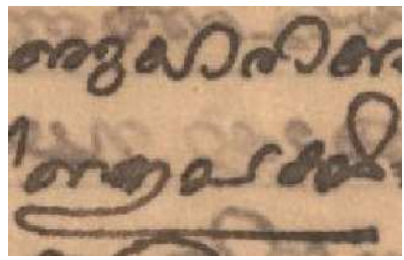

(a) Input

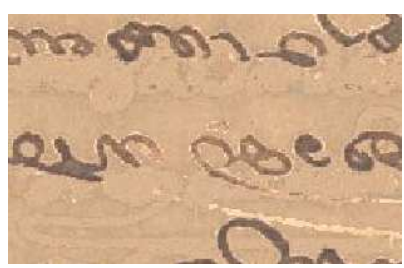

(c) Bleed Layer

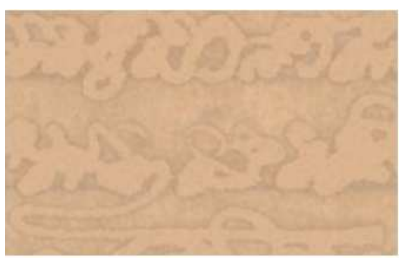

(b) BG Layer

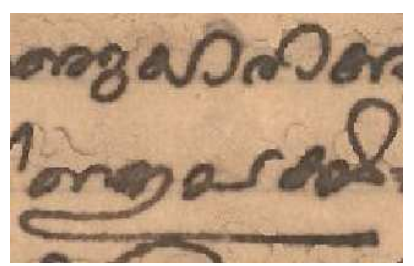

(d) Ink Layer
Fig. 1. A document image (a) that is decomposed into (b) background, (c) ink-bleed, and (d) foreground ink layers.

There have been significant progress in automatic restoration of degraded documents in the past few years. Most of them deal with the problem of efficient removal of ink-bleed and of recovering most of the foreground strokes. The work can be broadly classified based on weather it uses the image of a single face or aligned images of both front and back sides. Initial works in the area [4], [5], [6] approached the problem as a segmentation problem using a single face and applied variants of local or global thresholding. These methods work well on cleaner images with limited amount of inkbleed. Drira et al. [7] presented an approach for targeting complex inkbleed by adaptive thresholding, where the dimensionality of an RGB image is first reduced via Principal Component Analysis(PCA) and the classification is done by an iterative clustering of PCA data into two groups. Tonazzini et al. [8] on the other hand used signal separation via Independent Component Analysis(ICA). It assumes a linear model and decomposes an RGB image into three signals corresponding to foreground, background and inkbleed. A recent work by Wolf [3] uses a two-layer MRF, where the MRF prior is separated for two different label fields. The two MRF priors allows them to do the separation between the verso pixels covered by recto pixels and verso pixels not covered by recto pixels.

Non-blind separation where images from both sides of a document is used requires an initial alignment of these images. Wang et al. [9] and Tan et al. [10] used wavelet based approaches to help improve the thresholding of inkbleed pixels. Both of these approaches used a global alignment to compute the corresponding pixels of the two images and hence suffered 
from the imperfect overlay of the two images. Sharma [11] aligned the two images using image registration techniques. He used a reflectance model by taking into account a bleed through spread function. He then approximated and corrected this model with an adaptive linear filter. Tonazzini et al. [12] introduced a two-layer MRF model based on the linear mixture model and used an EM algorithm for estimating the inverse of the mixing matrix. They also proposed another model that uses blind signal separation to restore original images by using the same linear mixture model [13]. The major drawback of their approach is the linearity assumption in the model.

A recent work by Huang et al. [1] simultaneously reduced ink-bleed in both the recto and verso images. They used a user-assisted approach to mark few foreground, background and ink-bleed pixels and then used a dual-layer MRF setup for classification. The approach does not require any assumption about the ink-bleed intensity or extensive parameter tuning. They also used an additional local alignment process to align the two images more accurately. Moghaddam et al. [14] also reduced the ink-bleed simultaneously by using three diffusion models one each for recto-foreground, verso-foreground and background. For the information about the background he uses the scan of real paper without any text and his model relies on five user-tuned parameters. Lu et al. [2] extended the work of [1] by directing the user to provide markup where it is needed. They collect an small amount of user markup and classifies the image. This process is iterated for the pixels classified with low-confidence. By the use of user-assistance they were able to produce good results even for the difficult examples with strong ink-bleed. A more recent work by Hanasusanto et al. [15] used Chan-Vese active contour model [16] to incorporate information from the front and back sides of the ink-bleed document. They used functional minimization method for ink-bleed removal and used it again to restore broken foreground strokes. The approach is able to deal with strong ink-bleed overlaps with foreground strokes. However they do not label the recto and verso images simultaneously.

We aim to go beyond detection and removal of ink-bleed and attempt to restore the document images to a time where the document was just written. This requires us to model degradations in ink, paper and bleed separately. Hence, instead of treating the problem as that of classifying each pixel as belonging to either of foreground, background or ink-bleed, we aim to find the contribution of background, ink and bleedthrough at each pixel. We assume that color value at each pixel is the result of some combination of background, foreground ink and ink-bleed from the back side of the document. We propose a document image formation model that allows us to separate and deal with each of the above components and a degradation model that predicts how it might have changed over the period of time and how to restore it back to its original state.

\section{Document Image Model}

This section discusses the complete document image model, we begin with the discussion of document generation, here we discuss the process that goes when we write something over a paper, how the ink gets absorbed and how the background of a paper affects the intensity values. This state of the document that gets generated is something that we want to recover. In Document degradation model we discuss the several degradation processes that happens over a period of time and how the intensity values of each pixel undergoes changes.

\section{A. Document Generation Model}

If we go back to the time when the text was actually being written on the paper, then initially the paper was blank and for every pixel we only had the $B g$ value. This is the original $B g$ value without any degradation or any application of ink or ink-bleed. Now as the text is written over this paper by the application of ink, then based on the ink properties various changes occurs to the color values of these pixels. Some of these changes are instantaneous and some occur over a period of time. The immediate effect that is noticed is the visibility of the ink. The ink's opacity describes how opaque or transparent an ink is and to what degree the ink allows or prohibits the transmission of light through it and how well the background on which the ink has been printed can be seen. It is this property of the ink which determines the effect of the background on the color values of ink pixels. Now our paper will have some ink pixels and the rest are the original $B g$ pixels. Lets call the intensity values at these original $i n k$ and $B g$ pixels as $i n k^{\prime}$ and $B g^{\prime}$. The color values of these ink pixels will lie in a range depending on the amount of ink applied, how much the ink penetrates into the paper and the paper's thickness. Here we are assuming that the ink won't penetrate enough to become visible on the opposite side and this process will happen at some later time. Lets define $I^{\prime}$ as the intensity referring to the Ink applied. We can define the intensity of $i n k$ at each pixel $p$ as:

$$
\operatorname{In} k_{p}^{\prime}=I^{\prime \alpha_{p}^{\prime}} \cdot B g_{p}^{\prime}
$$
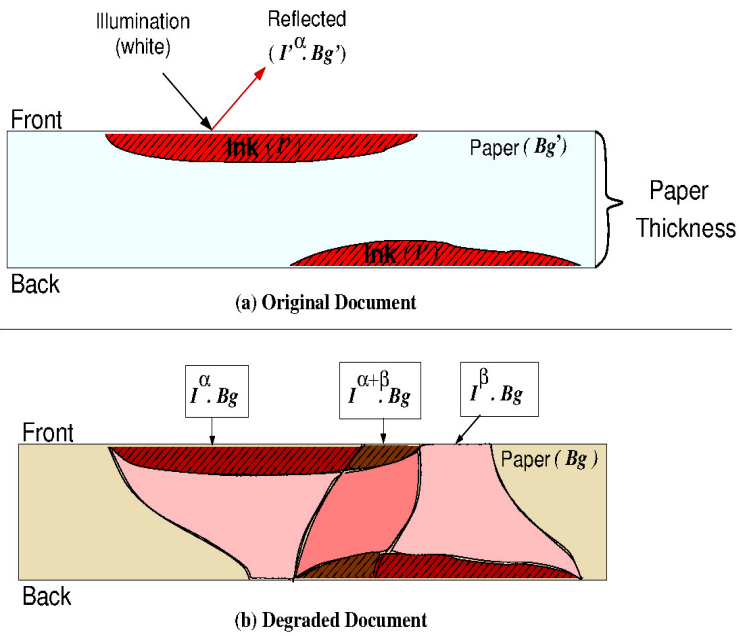

Fig. 2. (a) Document generation model: The combination of paper color with ink is modeled $I^{\prime \alpha} . B G^{\prime}$. The degradation process (b) creates bleed of ink from one face to another and also changes the color of ink and paper.

Here, $\alpha_{p}^{\prime}$ is defined for each pixel $p$ and it refers to the amount of ink present at each pixel. Most of the ancient documents are written by the use of a quill, the ink intensity is highest at the first point of contact and then it keeps on decreasing until the quill is dipped again in the ink. Here we have defined $\alpha$ at each pixel to capture this phenomena. Note that $I^{\prime}$ remains constant for all the pixels, based on 
the assumption that the ink is not changed while writing a complete document. For $B g$ pixels $\alpha^{\prime}=0$ and for Ink pixels $\alpha^{\prime}$ can lie in a range $(0,1]$.

\section{B. Document Degradation Model}

To achieve a degraded document image restoration of acceptable quality, a better understanding of the different types of image degradation processes is required.

The permanence of an ink pigment is the property which determines the extent to which an ink will retain its color strength and brightness with time or upon the exposure to light. This property of ink will change the value of $I^{\prime}$ over time. With the passage of time and due to variety of other factors including the ink's chemical makeup, the paper's physical and chemical construction and the amount of humidity in the environment, the value of $\alpha^{\prime}$ will decrease over time. Due to the ageing of the document, the paper also undergoes changes, it tends to become brittle and the brightness of the paper decreases. Various chemical reactions and the oxidation of the cellulose results in the yellowing of the paper. Due to all these reasons the $B g^{\prime}$ value also changes over time. Lets remove superscripts for the new values of $\alpha, B g, I n k$ and $I$. Now for every pixel $p$, we have

$$
\operatorname{In} k_{p}=I^{\alpha_{p}} \cdot B g_{p}
$$

One more prominent phenomena that will happen over time is that the ink will seep through the paper to interfere with the opposite side of the paper. In our generation model we only had background pixels and ink pixels. Now after the process of degradation we will have background pixels, ink pixels and some ink-bleed pixels.

For the rest of this paper lets define $p$ and $p^{\prime}$ to denote front image pixel and the corresponding back image pixel, $q$ and $q^{\prime}$ will be used to denote the pixels corresponding to $4-\mathrm{N}$ neighborhood of $p$ and $p^{\prime}$ respectively.

After taking ink-bleed into consideration the equation 2 will become

$$
\operatorname{In} k_{p}=I^{\alpha_{p}} \cdot B g_{p} \cdot I^{\beta_{p}}
$$

Here, $\beta$ refers to the amount of ink that is coming from the other side of the paper as a result of ink-bleed. Theoretically, the value of $\beta$ can lie in a range of $[0,1]$ and its magnitude tells about the severity of ink-bleed in the document. Note that the value of $\beta_{p}$ depends on the value of $\alpha_{p^{\prime}}$ and similarly the $\alpha_{p}$ influences the value of $\beta_{p^{\prime}}$. It's this $\operatorname{In} k_{p}$ that we observe from the input images and from this we want to get an estimate of $I n k_{p}^{\prime}$. Figure 2 explains the complete process of document degradation in detail.

\section{ESTIMATION OF DEGRADATION}

In this Section we present a detailed overview about the complexity of the problem, and the assumptions taken in order to do the estimation.

\section{A. Complexity of the problem}

From section II we know that $\alpha, \beta \in[0,1]$. Estimating the values of $\alpha$ and $\beta$ for each pixel is essentially a continuous class labelling problem with infinite number of classes where each $\alpha$ and $\beta$ can take any value from zero to one. All the existing techniques that we have seen in Section I, more or less tries to label each pixel as either a foreground, background or an ink-bleed pixel. Our problem can be solved by defining an MRF over the image pixels and posing the problem as a piecewise continuous restoration task, wherein the single-site and pair-site clique potential functions are defined. The details of which can be found at [17]. We simplify this problem and define a discrete set of values for $\alpha$ and $\beta$. The details of which is given in section III-B4.

\section{B. Details of Algorithm}

1) Input and Pre-processing: The input to our system is two high resolution images $(2 \mathrm{KX} 2 \mathrm{~K})$ corresponding to the front and the back of a page. These images need not be aligned and in addition may also suffer from 3D surface variations. Global and Local alignment of images are done as is described in [1].

2) Background vs Non-Background Classification: Once the images are aligned, we classify all the pixels as either a background pixel or a non-background pixel. For this we take assistance from the user and few background and nonbackground(foreground + ink-bleed) pixels are marked by the users. Two features $C_{p}$, and $\rho_{p}$ are used to train an SVM classifier. Here $C_{p}$ refers to grayscale pixel intensity of the front image pixels and $\rho_{p}$ is defined as the ratio of intensities $\frac{C_{p}}{C_{p}}$. Huang et al. [1] used the ratio feature alone for the initial classification of pixels as foreground, inkbleed and background based on the assumption that the ratio value for foreground data is mainly distributed between zero and one, inkbleed larger than one and background is mainly around one. This approach classifies foreground data with ratio values close to one as background. Whereas the use of ratio features along with the intensity values classifies the background and nonbackground(foreground + inkbleed) data quite robustly. We used libsvm [18] with the Radial Basis Kernel.

3) Background and Ink Estimation: For all the pixels labelled as non-background, we try to estimate the expected background value at these points. This process can be called as background generation, which in itself is an ill-posed problem as the exact RGB composition for the background is completely lost. We used the patch based approach to estimate the background pixels. A small patch is taken from the background region with similarities to a patch around the target pixel and thereby approximating the background values. The ink that we observe is the $I n k_{p}$ of equation 3 , from this we want to get an estimate of $I$. For this we took the help of user-assistance and marked few strongest Ink pixels such that the corresponding back image pixels does not belong to foreground pixels. We took the average of all these user marked pixels as an estimate of $I$.

4) MRF Formulation: After the initial classification of each pixel as either a background pixel or non-background pixel, we need to estimate the value of $\alpha$ and $\beta$ for each non-background pixel $p$, corresponding to the intensity of ink and bleed at that pixel. Instead of dealing with the continuous values of $\alpha$ and $\beta$, we release the assumption of continuity and define discrete values so that $\alpha \in\{0,0.25,0.50,0.75,1\}$ and $\beta \in$ $\{0,0.25,0.50,0.75\}$. Here we are assuming that $\beta$ can never 
take the value of one. Note that it doesn't mean that bleed is always lighter than the foreground strokes, it only suggests that bleed can never be equal to the strongest foreground strokes, there can be cases where $\beta>\alpha$. Now we have 20 combinations of $\langle\alpha, \beta\rangle$ that can be associated to each image pixel. Lets define our label set as $\mathcal{L}=\left\{l_{1}, l_{2}, \ldots, l_{20}\right\}$, corresponding to 20 different combinations of $\langle\alpha, \beta\rangle$ and $l^{p} \in \mathcal{L}$ is the label assigned to pixel $p$. With these discrete definitions of $\alpha$ and $\beta$, the problem can now be formulated as a discrete labeling MRF where each pixel, $p$ is assigned some combination of $\langle\alpha, \beta\rangle, l^{p}$. Our implementation of MRF is motivated by the work of Huang et al. [1], where instead of defining MRF for a trivial task of labelling foreground, background and ink-bleed, a more complex set of labels $\mathcal{L}$ is modeled. The optimal label assignment of an MRF is found by minimization of below energy function:

$$
E=E_{d}+\phi E_{s},
$$

where $E_{d}$ is the energy associated with the data-terms, which is the likelihood of assigning a label $l^{p} \in \mathcal{L}$ to each pixel, $p$ and $E_{s}$ is the energy associated with the smoothness term, which is the cost of assigning different label values to the neighboring pixels. The scalar weight $\phi$ is to balance the two terms. The readers are directed to [17] for the details about MRF formulation. We define Dual Layer MRF similar to [1] and the details about intra-layer and inter-layer edges can be seen at [1]. The dual layer MRF is chosen due to its effectiveness in preserving the foreground strokes. The details about the data term and smoothness term is given below.

Data Term $E_{d}$, The definition for data cost $E_{d}$ is same for both the front and back image. Using the estimation of background and $i n k$ from section III-B3 and using equation 3, the value of $\alpha+\beta$ can be calculated as

$$
\alpha_{p}+\beta_{p}=\log _{I}\left(\frac{\operatorname{In} k_{p}}{B g_{p}}\right)
$$

Lets define $\lambda_{p}=\left(\alpha_{p}+\beta_{p}\right)$. A 3-dimensional feature vector with values $<\lambda_{p}, \lambda_{p^{\prime}},\left|\lambda_{p}-\lambda_{p^{\prime}}\right|>$ is defined, where $|a-b|$ gives the absolute difference of two values $a$ and $b$. This 3dimensional feature vector is capable of capturing the amount of ink present in the front and the back side of the page and their differences. Using these features a set of twenty SVM classifiers are trained corresponding to twenty different classes based on one-against-rest method. To get training samples in order to train these SVMs we carefully generated synthetic data corresponding to these twenty classes.

For the generation of synthetic data, we downloaded two images of handwritten text and two background images. Text is first rendered over the background images and then the different sets of synthetic images are generated by varying the values of $\alpha$ and $\beta$ of equation 3 .

Each pixel $p$ is then classified based on these SVMs and its similarities (or probabilities) $S_{i}(i$ going from one to twenty) are calculated similar to [1]. The data cost, $E_{d}$, for each label is defined as:

$$
E_{d}\left(l^{p}=l_{i}\right)=\frac{\sum_{j=1}^{20} S_{j}-S_{i}}{19 * \sum_{j=1}^{20} S_{j}}
$$

Note that the data cost, $E_{d}$, from Eq 6 lies in a range of zero to one, and $\sum_{i=1}^{20} E_{d}\left(l^{p}=l_{i}\right)=1$.

Smoothness Term $E_{s}$, In the dual-layer MRF setup, each pixel, $p$ have edges with its 4-N neighborhood (Intra-layer edges) and there is also an edge with the corresponding back page pixel $p^{\prime}$ (Inter-layer edge). The same setup is defined for both the front and back image. We can write the smoothness term as :

$$
E_{s}=\sum_{p, q \in \mathcal{N}} V_{1}\left(l^{p}, l^{q}\right)+\sum_{p, p^{\prime} \in \mathcal{M}} V_{2}\left(l^{p}, l^{p^{\prime}}\right)
$$

Here $V_{1}$ corresponds to intra-layer edge costs and $V_{2}$ corresponds to inter-layer edge costs, both of these terms are weighted equally.

Intra-Layer Edge Costs: The intra-layer edge costs are defined as:

$$
\begin{aligned}
& V_{1}\left(l^{p}=<\alpha_{p}, \beta_{p}>, l^{q}=<\alpha_{q}, \beta_{q}>\right)= \\
&\left|\alpha_{p}-\alpha_{q}\right|^{2}+\left|\beta_{p}-\beta_{q}\right|^{2}
\end{aligned}
$$

These costs just enforce smoothness constraints to neighboring pixels, assigning more cost to the neighboring pixels with large variations in their corresponding values of $\alpha$ and $\beta$.

Inter-Layer Edge Costs Inter-layer edge costs, $V_{2}\left(l^{p}, l^{p^{\prime}}\right)$, are defined as:

$$
V_{2}\left(l^{p}=<\alpha_{p}, \beta_{p}>, l^{p^{\prime}}=<\alpha_{p^{\prime}}, \beta_{p^{\prime}}>\right)=\delta_{\alpha_{p} \beta_{p^{\prime}}}+\delta_{\alpha_{p^{\prime}} \beta_{p}}
$$

where $\delta_{\alpha \beta}$ is defined in the following table:

\begin{tabular}{|c|c|c|c|c|c|}
\hline \multirow{2}{*}{$\beta$} & \multicolumn{5}{|c|}{$\alpha$} \\
\cline { 2 - 6 } & 0 & 0.25 & 0.5 & 0.75 & 1 \\
\hline 0 & 0 & $\omega / 4$ & $\omega / 3$ & $\omega / 2$ & $\omega$ \\
\hline 0.25 & $\infty$ & $\infty$ & $\omega / 4$ & $\omega / 3$ & $\omega / 2$ \\
\hline 0.5 & $\infty$ & $\infty$ & $\infty$ & $\omega / 4$ & $\omega / 3$ \\
\hline 0.75 & $\infty$ & $\infty$ & $\infty$ & $\infty$ & $\omega / 4$ \\
\hline
\end{tabular}

In the above table, we are enforcing the constraint that the intensity of bleed at any pixel $p$ is always less than the ink intensity at pixel $p^{\prime}$, thereby assigning infinite cost to all these cases. As the value of $\alpha$ for a pixel $p$ increases, this in turn increases the likelihood for a pixel $p^{\prime}$ to have a higher value of $\beta$, we have captured this notion by assigning an increasing cost for the cases when the value of $\beta$ at pixel $p^{\prime}$ is relatively less compared to the value of $\alpha$ at pixel $p$.

The only two parameters in our MRF formulation are $\phi$ and $\omega$. For our current work, we have empirically fixed the values of $\phi$ and $\omega$ as one.

Minimizing the Energy Function For minimizing the energy function we used the $\alpha$-expansion move of graph cuts [19]. The dual layer MRF model is implemented by modifying the Middlebury's MRF code provided by [20]. In all of our experiments, energy minima is reached within four or five iterations. 


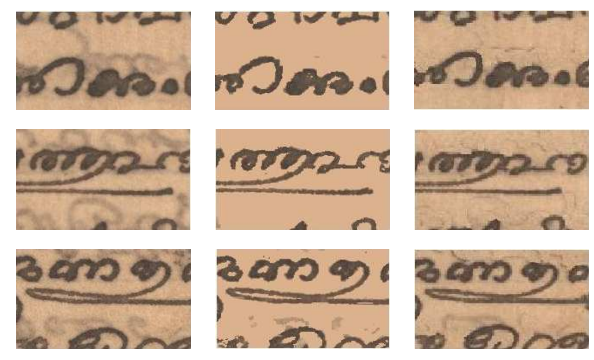

Fig. 3. Comparison of DL-MRF-SVM of [1] and the proposed method(From left to right input image, DL-MRF-SVM and proposed method). The proposed method is able to preserve the background and the finer details of foreground.

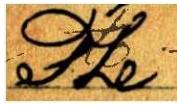

(a) $\alpha=1, \beta=.25$

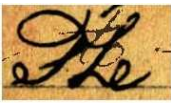

(b) $\alpha=.8, \beta=.3$

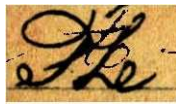

(c) $\alpha=1, \beta=1$

Fig. 4. Synthetic dataset. For 4(a) the algorithm gave correct values of $\alpha$ and $\beta$, For 4(b) $\alpha=0.75$ and $\beta=0.25$ were reported by the algorithm. The proposed algorithm failed for $4(\mathrm{c})$, it considered everything as the foreground.

\section{RESUlts And AnAlysis}

We ran our experiments over a dataset of Malayalam handwritten manuscripts, these documents are mostly 150 years old and contains the conversations between the king and the local groups. We compared our approach with the DLMRF-SVM of [1], which has shown better results than the previous approaches.

One of the major contribution of this paper is an attempt to preserve the background information, and to extract the bleed even from the foreground strokes. We can see from the Figure 3 that our approach was able to recover the document in a better state than the corresponding DL-MRF-SVM of [1]. Figure 4 shows the evaluation on synthetic data and the failure case of our algorithm. Infact our algorithm fails everytime the value of $\beta$ goes above 0.85 .

As their is no ground truth available for the images, we manually evaluated the corresponding results and error is calculated as the number of foreground words with undetected or missing strokes. We evaluated our results over a set of twenty images and our method had a precision accuracy of 93.4, where as the DL-MRF-SVM gave an accuracy of 89.4. Precision is defined as $\left(W-W^{F}\right) /\left(W+W^{B}\right)$, where $W$ is the total number of foreground words, $W^{F}$ is the number of incorrectly classified foreground words, and $W^{B}$ are the number of stroke-size background or ink-bleed regions that were classified as foreground.

\section{CONCLUSION}

Our results demonstrate that our model can generate results with the view of keeping the finer details preserved, we were able to preserve most of the foreground strokes and the background estimation is also of acceptable quality. Though there can be still better methods to get an estimation of background and we are keeping this as a future work. We require user markup for the initial classification of background vs non-background pixels and again for the estimation of ink, and in accordance with most of the supervised learning approaches, we assume that the user labelled data is correct.

\section{REFERENCES}

[1] Y. Huang, M. Brown, and D. Xu, "A framework for reducing ink-bleed in old documents," in Computer Vision and Pattern Recognition, 2008. CVPR 2008. IEEE Conference on. IEEE, 2008, pp. 1-7.

[2] Z. Lu, Z. Wu, and M. Brown, "Directed assistance for ink-bleed reduction in old documents," in Computer Vision and Pattern Recognition, 2009. CVPR 2009. IEEE Conference on. IEEE, 2009, pp. 88-95.

[3] C. Wolf, "Document ink bleed-through removal with two hidden markov random fields and a single observation field," Pattern Analysis and Machine Intelligence, IEEE Transactions on, vol. 32, no. 3, pp. 431-447, 2010.

[4] J. Bescos, "Image processing algorithms for readability enhancement of old manuscripts," in Proc. Intl Electronic Imaging Exposition and Conf, 1989, pp. 392-397.

[5] F. e. a. Mintzer, "Toward on-line, worldwide access to vatican library materials," IBM Journal of Research and Development, vol. 40, no. 2, pp. 139-162, 1996.

[6] Z. Shi and V. Govindaraju, "Historical document image enhancement using background light intensity normalization," in Pattern Recognition, 2004. ICPR 2004. Proceedings of the 17th International Conference on, vol. 1. IEEE, 2004, pp. 473-476.

[7] D. Fadoua, F. Le Bourgeois, and H. Emptoz, "Restoring ink bleedthrough degraded document images using a recursive unsupervised classification technique," Document Analysis Systems VII, pp. 38-49, 2006.

[8] A. Tonazzini, L. Bedini, and E. Salerno, "Independent component analysis for document restoration," International Journal on Document Analysis and Recognition, vol. 7, no. 1, pp. 17-27, 2004.

[9] Q. Wang, T. Xia, L. Li, and C. Tan, "Document image enhancement using directional wavelet," in Computer Vision and Pattern Recognition, 2003. Proceedings. 2003 IEEE Computer Society Conference on, vol. 2. IEEE, 2003, pp. II-534.

[10] C. Tan, R. Cao, and P. Shen, "Restoration of archival documents using a wavelet technique," Pattern Analysis and Machine Intelligence, IEEE Transactions on, vol. 24, no. 10, pp. 1399-1404, 2002.

[11] G. Sharma, "Show-through cancellation in scans of duplex printed documents," Image Processing, IEEE Transactions on, vol. 10, no. 5, pp. 736-754, 2001.

[12] A. Tonazzini, L. Bedini, and E. Salerno, "A markov model for blind image separation by a mean-field em algorithm," Image Processing, IEEE Transactions on, vol. 15, no. 2, pp. 473-482, 2006.

[13] A. Tonazzini, E. Salerno, and L. Bedini, "Fast correction of bleedthrough distortion in grayscale documents by a blind source separation technique," International Journal on Document Analysis and Recognition, vol. 10, no. 1, pp. 17-25, 2007.

[14] R. Moghaddam and M. Cheriet, "Low quality document image modeling and enhancement," International journal on document analysis and recognition, vol. 11, no. 4, pp. 183-201, 2009.

[15] G. Hanasusanto, Z. Wu, and M. Brown, "Ink-bleed reduction using functional minimization," in Computer Vision and Pattern Recognition (CVPR), 2010 IEEE Conference on. IEEE, 2010, pp. 825-832.

[16] T. Chan and L. Vese, "Active contours without edges," Image Processing, IEEE Transactions on, vol. 10, no. 2, pp. 266-277, 2001.

[17] S. Li, Markov random field modeling in image analysis. Springer, 2009.

[18] C. Chang and C. Lin, "Libsvm: a library for support vector machines," ACM Transactions on Intelligent Systems and Technology (TIST), vol. 2, no. 3, p. 27, 2011.

[19] Y. Boykov and V. Kolmogorov, "An experimental comparison of mincut/max-flow algorithms for energy minimization in vision," Pattern Analysis and Machine Intelligence, IEEE Transactions on, vol. 26, no. 9, pp. 1124-1137, 2004.

[20] R. Szeliski, R. Zabih, D. Scharstein, O. Veksler, V. Kolmogorov, A. Agarwala, M. Tappen, and C. Rother, "A comparative study of energy minimization methods for markov random fields," Computer VisionECCV 2006, pp. 16-29, 2006. 